\title{
OPTIMIZATION OF THE SERVICE STRATEGY IN A QUEUEING SYSTEM WITH ENERGY HARVESTING AND CUSTOMERS' IMPATIENCE
}

\author{
Alexander DUdin ${ }^{a}$, MoOn Ho LEE $^{b, *}$, Sergey DUdiN $^{a}$ \\ ${ }^{a}$ Department of Applied Mathematics and Computer Science \\ Belarusian State University, 4, Nezavisimosti Ave., Minsk, 220030, Belarus \\ e-mail: dudin@bsu.by, dudin85@mail.ru \\ ${ }^{b}$ Division of Electronics \\ Chonbuk National University, Jeonju, 561-765, South Korea \\ e-mail: moonho@jbnu.ac.kr
}

\begin{abstract}
A single-server queueing system with an infinite buffer is considered. The service of a customer is possible only in the presence of at least one unit of energy, and during the service the number of available units decreases by one. New units of energy arrive in the system at random instants of time if the finite buffer for maintenance of energy is not full. Customers are impatient and leave the system without service after a random amount of waiting time. Such a queueing system describes, e.g., the operation of a sensor node which harvests energy necessary for information transmission from the environment. Aiming to minimize the loss of customers due to their impatience (and maximize the throughput of the system), a new strategy of control by providing service is proposed. This strategy suggests that service temporarily stops if the number of customers or units of energy in the system becomes zero. The server is switched off (is in sleep mode) for some time. This time finishes (the server wakes up) if both the number of customers in the buffer and the number of energy units reach some fixed threshold values or when the number of energy units reaches some threshold value and there are customers in the buffer. Arrival flows of customers and energy units are assumed to be described by an independent Markovian arrival process. The service time has a phase-type distribution. The system behavior is described by a multi-dimensional Markov chain. The generator of this Markov chain is derived. The ergodicity condition is presented. Expressions for key performance measures are given. Numerical results illustrating the dependence of a customer's loss probability on the thresholds defining the discipline of waking up the server are provided. The importance of the account of correlation in arrival processes is numerically illustrated.
\end{abstract}

Keywords: energy harvesting, threshold strategy, optimization, Markovian arrival process.

\section{Introduction}

Queueing theory has wide applications in capacity planning, performance evaluation and optimization of a huge variety of real life objects and processes. Traditional queueing models assume that there is a flow of customers which is processed by some server (in single-server settings) or servers (in multi-server settings). However, in some real life systems the situation is more complicated because the server is not an independent object. Its operation depends on the presence of some additional items (windows, tokens, permissions, details, semi-finished products, etc.).

${ }^{*}$ Corresponding author
The standard situation considered in the literature devoted to analysis of queueing systems with additional items is as follows. The total number of additional items in the system is finite and the items are reusable. They are "borrowed" by the server to provide service to a customer. After service completion, the item returns to the pool of items. Such a scenario takes place, e.g., when it is necessary to regulate the speed of customers admission (in protocols like the sliding window, the leaky bucket, etc.) or when service is provided with the help of some equipment. Among related papers, we can mention the one by Dudin and Klimenok (1996), where the additional item is interpreted as a passive server, and that by Kim et al. (2009), where the additional item is interpreted as a 
token that is necessary to start the service of a session.

In this paper, we consider another situation. We also assume that the service of a customer requires the use of an additional item, but this item is not reusable. It disappears from the system forever after the beginning of service of the customer. If during a service completion epoch there is no additional item in the system, the service of customers is suspended. New additional items are delivered into the system at random instances. We suggest that the service is resumed when the number of additional items and (or) the number of customers exceeds some preassigned thresholds. Generally speaking, several additional items are used at the service resume moment. Customers are impatient and leave the system (are lost) after a random amount of time. The goal is to assign the values of thresholds aiming to minimize customer loss probability.

Examples of possible applications of the model under consideration are as follows.

(i) Optimization of operation of a sensor node. For more information about the concept of sensor networks and related research, see the work of Akyildiz et al. (2002). The sensor node senses a random field and generates packets to be transmitted to a central node. The sensor node has a battery of a finite capacity and harvests energy during its operation from outside (we can mention solar cells, wind turbines, piezo electric cells, radio frequency collectors, etc.). We assume that the energy is slotted to energy units and one unit is required to transmit one packet. To save energy, the node switches to sleep mode after all available packets are transmitted and stays in this mode for some time. This time should be long enough to earn energy units for battery for future transmissions, but it should be short enough due to obsolescence of information collected by the sensor node.

If the waiting time of a packet exceeds some level, transmission of this packet becomes meaningless. The queueing model under study should help to find some trade-off in this situation. Analogous models were formulated, e.g., in the papers of Yang and Ulukus (2012a), Sharma et al. (2010), Tutuncuoglu and Yener (2012) or Yang and Ulukus (2012b), where more information about quite extensive literature concerning systems with energy harvesting is presented. However, the analysis was provided there either in deterministic settings or not in terms of queueing theory.

Here we provide queueing analysis of the corresponding system. We assume that arrival flows of customers and energy units are random and the service time of a customer is random as well. Recently, an analysis of a queueing model with energy harvesting was performed by Gelenbe (2015), who assumed that the service to customers is performed instantaneously. This implies that the buffer of customers or (and) the buffer of energy should be always empty. We assume that the service time is random and not equal to zero.

We do not follow the traditional assumptions that the arrival flows are stationary Poisson and the service time distribution is exponential. We assume that the arrival flows of customers (packets) and units are described by a Markovian arrival process. This allows us to catch possible correlation in arrival processes (possibility to have time intervals when customers or energy units arrive more or less frequently). This is important from the point of view of applications because, e.g., the speed of energy harvesting may essentially vary depending on sunshine or speed of the wind. Concerning the service process, we assume that the service time has a $P H$ (phase-type) distribution, which is more general than an exponential distribution. As will be shown in numerical results below, correlation in arrival processes and variation of the service process drastically change the system characteristics comparing to those in the system with stationary Poisson arrival processes having the same mean arrival rate and an exponential service time distribution.

(ii) Performance evaluation of operation of a manufacturing station where the repairing of some devices is implemented. Repairing a device requires using return some semi-finished product. If this product is absent at the stock, the repair is not possible. In this case, it makes sense to offer vacation to the repairman and optimally choose the strategy of this repairman's from vacation.

The queueing model considered is the one with server vacations. However, it is not imbedded to some known class of vacation queueing models. Vacation models are usually classified (see, e.g., Doshi, 1986; Takagi, 1991; Tian and Zhang, 2006), by the discipline of switching the server off (exhaustive, gated, limited, etc.) and switching it on ( $N$-, $T$-, $E$-strategies, etc.). The model under study is close in some sense to systems with exhaustive service (service is stopped after the system becomes empty), but indeed service here is not exhaustive. It may be interrupted also due to the lack of additional units. This model is close to systems with limited service, but models considered in the literature do not assume that the limit depends on future dynamics of service, while we here have such dependence. The model considered is close to systems with $N$-strategy of switching the server on. However, here we suggest alternative schemes for switching the server on, depending not only on the number of customers in the queue, but also on the current number of available additional units.

The remainder of this paper is structured as follows. In Section 2, the mathematical model is described. In Section 3 , the process of the system states is completely defined. The ergodicity condition and the stationary probabilities of the system states are discussed in Section 4. Formulas for computation of the performance 
measures of the system are presented in Section 5 . Numerical examples are given and an optimization problem is discussed in Section 6. Section 7 concludes the paper.

\section{Mathematical model}

We consider a single-server queueing system with an infinite buffer for customers and a buffer of capacity $K$ for energy units. The structure of the system under study is presented in Fig. 1.

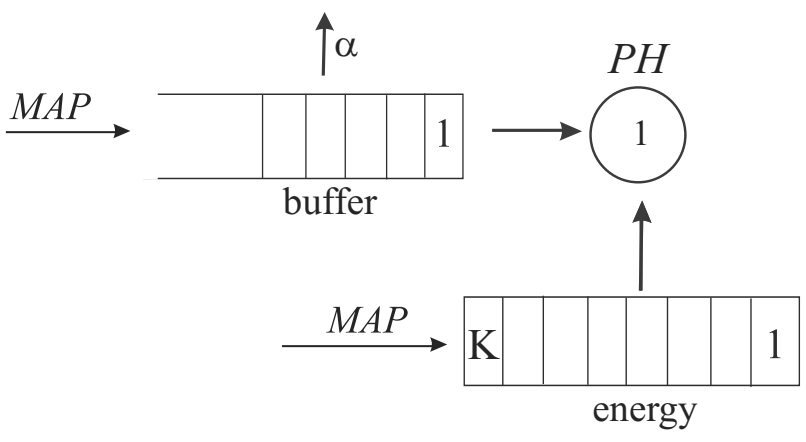

Fig. 1. Queueing system under study.

Customers arrive in the system according to a Markovian arrival process. We will code this process as $M A P_{1}$. Arrivals in $M A P_{1}$ are directed by an irreducible continuous time Markov chain $\nu_{t}, t \geq 0$, with the finite state space $\{0,1, \ldots, W\}$. The sojourn time of the Markov chain $\nu_{t}, t \geq 0$, in the state $\nu$, has an exponential distribution with the parameter $\lambda_{\nu}, \nu=\overline{0, W}$. Here, notation such as $\nu=\overline{0, W}$ means that $\nu$ assumes values from the set $\{0,1, \ldots, W\}$. After this sojourn time expires, with probability $p_{k}\left(\nu, \nu^{\prime}\right)$, the process $\nu_{t}$ transits to the state $\nu^{\prime}$ and $k$ customers, $k=0,1$, arrive in the system. The intensities of transitions from one state to another, which are accompanied by arrival of $k$ customers, are combined to the square matrices $D_{k}, k=0,1$, of size $W+1$. The matrix generating function of these matrices is $D(z)=D_{0}+D_{1} z,|z| \leq 1$. The matrix $D(1)$ is an infinitesimal generator of the process $\nu_{t}, t \geq 0$. The stationary distribution vector $\boldsymbol{\theta}$ of this process satisfies the system of equations $\boldsymbol{\theta} D(1)=\mathbf{0}, \boldsymbol{\theta} \mathbf{e}=1$. Here and in the sequel $\mathbf{0}$ is a zero row vector and $\mathbf{e}$ denotes a unit column vector. If the dimensionality of a vector is not clear from the context, it is indicated as a lower index, e.g., $\mathbf{e}_{\bar{W}}$ denotes the unit column vector of dimensionality $\bar{W}=W+1$.

The average intensity $\lambda_{c}$ (fundamental rate) of the $M A P_{1}$ is defined by $\lambda_{c}=\boldsymbol{\theta} D_{1} \mathbf{e}$. The variance $v$ of customers' inter-arrival time is calculated by

$$
v=2\left(\lambda_{c}\right)^{-1} \boldsymbol{\theta}\left(-D_{0}\right)^{-1} \mathbf{e}-\left(\lambda_{c}\right)^{-2},
$$

the squared coefficient of the variation is calculated by

$$
c_{\mathrm{var}}=2 \lambda_{c} \boldsymbol{\theta}\left(-D_{0}\right)^{-1} \mathbf{e}-1,
$$

while the coefficient of correlation of intervals between successive arrivals is given by

$$
c_{\mathrm{cor}}=\frac{1}{v}\left(\left(\lambda_{c}\right)^{-1} \boldsymbol{\theta}\left(-D_{0}\right)^{-1} D_{1}\left(-D_{0}\right)^{-1} \mathbf{e}-\left(\lambda_{c}\right)^{-2}\right) .
$$

Units of energy arrive in the system according to the Markovian arrival process $M A P_{2}$. Arrivals in $M A P_{2}$ are directed by an irreducible continuous time Markov chain $\zeta_{t}, t \geq 0$, with the finite state space $\{0,1, \ldots, V\} . M A P_{2}$ is defined by the matrices $H_{0}$ and $H_{1}$. Let us denote the average intensity of $M A P_{2}$ as $\lambda_{e}$.

We assume that for service of one customer a unit of energy is required. So, when the customer is chosen for service, the number of units of energy decreases by one. If after the service completion epoch the buffer of customers or the buffer of energy is empty, the server is switched off. To switch the server on, $n, 0 \leq n<K$, units of energy are required. These units disappear from the buffer of energy at the moment of the server being switching on.

The server is switched on in the following two cases:

(i) the number of customers in the buffer is greater than or equal to $m, m \geq 1$, and the number of units of energy is greater than $k_{1}, n<k_{1}<K$;

(ii) the number of units of energy is greater than $k_{2}, k_{1} \leq$ $k_{2}<K$, and the buffer is not empty.

If, in the energy arrival epoch, the buffer of energy is full, then the arriving unit is lost.

The service time of a customer at the server has a $P H$ distribution with an irreducible representation $(\boldsymbol{\beta}, S)$. This service time can be interpreted as time until the underlying Markov process $\eta_{t}, t \geq 0$, with a finite state space $\{1, \ldots, M, M+1\}$ reaches the single absorbing state $M+1$ condition on the fact that the initial state of this process is selected from among the states $\{1, \ldots, M\}$ according to the probabilistic row vector $\boldsymbol{\beta}=$ $\left(\beta_{1}, \ldots, \beta_{M}\right)$. Transition rates of the process $\eta_{t}$ within the set $\{1, \ldots, M\}$ are defined by the sub-generator $S$, and transition rates into the absorbing state (which leads to service completion) are given by the entries of the column vector $\boldsymbol{S}_{0}=-S \mathbf{e}$.

The service time distribution function has the form $A(x)=1-\boldsymbol{\beta} e^{S x} \mathbf{e}$, the Laplace-Stieltjes transform $(L S T)$

$$
\int_{0}^{\infty} e^{-s x} \mathrm{~d} A(x)
$$

of this distribution is $\boldsymbol{\beta}(s I-S)^{-1} \boldsymbol{S}_{0}, \operatorname{Re} s>0$. The mean service time is calculated by $b_{1}=\boldsymbol{\beta}(-S)^{-1} \mathbf{e}$. The squared coefficient of variation is given by $c_{\mathrm{var}}=b_{2} / b_{1}^{2}-$ 1 , where $b_{2}=2 \boldsymbol{\beta}(-S)^{-2} \mathbf{e}$. 
The problem of fitting the measurements of arrival and service processes in real world systems with a Markovian arrival process and a $P H$ distribution can be solved by analogy with Mészáros et al. (2014).

Customers in the buffer can be impatient, i.e., the customer leaves the buffer after time exponentially distributed with the parameter $\alpha, 0 \leq \alpha<\infty$, after the arrival due to the lack of service.

\section{Process of the system states}

It is easy to see that the behavior of the system under study is described in terms of the following regular irreducible continuous-time Markov chain:

$$
\xi_{t}=\left\{i_{t}, r_{t}, k_{t}, \nu_{t}, \zeta_{t}, \eta_{t}\right\}, \quad t \geq 0,
$$

where, during the epoch $t, t \geq 0$,

- $i_{t}$ is the number of customers in the system, $i_{t} \geq 0$;

- $r_{t}$ is an indicator that indicates whether or not the server works: $r_{t}=0$ corresponds to the case when the server does not work and $r_{t}=1$ to the one when the server works;

- $k_{t}$ is the number of energy units, $k_{t}=\overline{0, K}$;

- $\nu_{t}$ is the state of the underlying process of $M A P_{1}$, $\nu_{t}=\overline{0, W}$;

- $\zeta_{t}$ is the state of the underlying process of $M A P_{2}$, $\zeta_{t}=\overline{0, V}$;

- $\eta_{t}$ is the state of the $P H$ service process, $\eta_{t}=\overline{1, M}$. The initial state of this process is defined, according to the stochastic vector $\boldsymbol{\beta}$, with service completion of the previous customer. When the server does not work, the chosen state is frozen.

The Markov chain $\xi_{t}, t \geq 0$, has the following state space:

$$
\begin{aligned}
& (\{0,0, k, \nu, \zeta, \eta\}, k=\overline{0, K}) \\
& \cup\left(\{i, 0, k, \nu, \zeta, \eta\}, i=\overline{1, m-1}, k=\overline{0, k_{2}}\right) \\
& \cup\left(\{i, 0, k, \nu, \zeta, \eta\}, i \geq m, k=\overline{0, k_{1}}\right) \\
& \cup(\{i, 1, k, \nu, \zeta, \eta\}, i \geq 1, k=\overline{0, K}) \\
& \nu=\overline{0, W}, \quad \zeta=\overline{0, V}, \quad \eta=\overline{1, M} .
\end{aligned}
$$

In the sequel, we use the following notation:

- $I$ is an identity matrix, and $O$ is a zero matrix of appropriate dimension;

- $\otimes$ and $\oplus$ indicate the symbols of a Kronecker product and a sum of matrices, respectively;
- $\bar{V}=V+1$

- $E^{+}$is a square matrix of size $K+1$ with all zero entries except $\left(E^{+}\right)_{l, l+1}, l=\overline{0, K-1}$, and $\left(E^{+}\right)_{K+1, K+1}$, which are equal to 1 ;

- $E_{k}^{+}, k=k_{1}, k_{2}$, is a square matrix of size $k+1$ with all zero entries except $\left(E_{k}^{+}\right)_{l, l+1}, l=\overline{0, k-1}$, which are equal to 1 ;

- $E^{-}$is a square matrix of size $K+1$ with all zero entries except $\left(E^{-}\right)_{l, l-1}, l=\overline{1, K}$, which are equal to 1 ;

- $E_{k}^{-}, k=k_{1}, k_{2}$, is a matrix of size $(k+1) \times(K+$ 1) with all zero entries except $\left(E_{k}^{-}\right)_{k, k-n}$, which is equal to 1 ;

- $\hat{I}_{k}, k=k_{1}, k_{2}$, is a matrix of size $(k+1) \times(K+1)$ with all zero entries except $\left(\hat{I}_{k}\right)_{0,0}$, which is equal to 1 ;

- $\bar{I}_{k}, k=k_{1}, k_{2}$, is a matrix of size $(K+1) \times(k+1)$ with all zero entries except $\left(\bar{I}_{k}\right)_{l, l}, l=\overline{0, k}$, which are equal to 1 ;

- $I_{k, j}$ is a matrix of size $(k+1) \times(j+1)$ with all zero entries except $\left(I_{k, j}\right)_{l, l}, l=\overline{0, \min \{k, j\}}$, which are equal to 1 ;

- $\tilde{I}$ is a matrix of size $\left(k_{2}+1\right) \times(K+1)$ with all zero entries except $(\tilde{I})_{l, l-n-1}, l=\overline{k_{1}+1, k_{2}}$, which are equal to 1 ;

- $I_{k}^{-}, k=k_{1}, k_{2}$, is the matrix of size $(K+1) \times$ $(K+1)$ with all zero entries except the entries $\left(I_{k}^{-}\right)_{k+1, l-n-1}, l=\overline{k+1, K}$, which are equal to 1 ;

- $\delta_{a=b}= \begin{cases}1, & \text { if } a=b \\ 0, & \text { otherwise }\end{cases}$

Let us enumerate the states of the Markov chain $\xi_{t}, t \geq 0$, in direct lexicographic order of the components $r, k, \nu, \zeta, \eta$, and refer to the set of the states of the chain having values $(i, r)$ of the first two components of the Markov chain as a macro-state $(i, r)$. Let $Q$ be the generator of the Markov chain $\xi_{t}, t \geq 0$, consisting of the blocks $Q_{i, j}$, which, in turn, consist of the matrices $\left(Q_{i, j}\right)_{r, r^{\prime}}$ of the transition rates of this chain from the macro-state $(i, r)$ to the macro-state $\left(j, r^{\prime}\right), r, r^{\prime}=0,1$. The diagonal entries of the matrices $Q_{i, i}$ are negative, and the modulus of the diagonal entry of the blocks $\left(Q_{i, i}\right)_{r, r}$ defines the total intensity of leaving the corresponding state of the Markov chain $\xi_{t}, t \geq 0$.

Theorem 1. The infinitesimal generator $Q=\left(Q_{i, j}\right)_{i, j \geq 0}$ of the Markov chain $\xi_{t}, t \geq 0$, has a block-tridiagonal 
structure:

$$
Q=\left(\begin{array}{ccccc}
Q_{0,0} & Q_{0,1} & O & O & \cdots \\
Q_{1,0} & Q_{1,1} & Q_{1,2} & O & \cdots \\
O & Q_{2,1} & Q_{2,2} & Q_{2,3} & \cdots \\
\vdots & \vdots & \vdots & \vdots & \ddots
\end{array}\right)
$$

The non-zero blocks $Q_{i, j}, i, j \geq 0$, have the following form:

$$
\begin{aligned}
& Q_{0,0}=I_{K+1} \otimes\left(D_{0} \oplus H_{0}\right) \otimes I_{M} \\
& +E^{+} \otimes I_{\bar{W}} \otimes H_{1} \otimes I_{M}, \\
& Q_{i, i}=\left(\begin{array}{cc}
Q_{i, i}^{(0,0)} & Q_{i, i}^{(0,1)} \\
O & Q_{i, i}^{(1,1)}
\end{array}\right), \quad i>0 \\
& Q_{i, i}^{(0,0)}=-i \alpha I+I_{k_{2}+1} \otimes\left(D_{0} \oplus H_{0}\right) \otimes I_{M} \\
& +E_{k_{2}}^{+} \otimes I_{\bar{W}} \otimes H_{1} \otimes I_{M}, \quad i=\overline{1, m-1}, \\
& Q_{i, i}^{(0,0)}=-i \alpha I+I_{k_{1}+1} \otimes\left(D_{0} \oplus H_{0}\right) \otimes I_{M} \\
& +E_{k_{1}}^{+} \otimes I_{\bar{W}} \otimes H_{1} \otimes I_{M}, \quad i \geq m, \\
& Q_{i, i}^{(0,1)}= \begin{cases}E_{k_{2}}^{-} \otimes I_{\bar{W}} \otimes H_{1} \otimes I_{M}, & i=\overline{1, m-1}, \\
E_{k_{1}}^{-} \otimes I_{\bar{W}} \otimes H_{1} \otimes I_{M}, & i \geq m,\end{cases} \\
& Q_{i, i}^{(1,1)}=I_{K+1} \otimes\left(D_{0} \oplus H_{0} \oplus S\right) \\
& +E^{+} \otimes I_{\bar{W}} \otimes H_{1} \otimes I_{M}-(i-1) \alpha I, \\
& i>0 \text {, } \\
& Q_{0,1}=\left(\begin{array}{ll}
Q_{0,1}^{(0,0)} & Q_{0,1}^{(0,1)}
\end{array}\right), \quad i>0 \\
& Q_{0,1}^{(0,0)}= \begin{cases}\bar{I}_{k_{1}} \otimes D_{1} \otimes I_{\bar{V} M}, & m=1, \\
\bar{I}_{k_{2}} \otimes D_{1} \otimes I_{\bar{V} M}, & m>1,\end{cases} \\
& Q_{0,1}^{(0,1)}= \begin{cases}I_{k_{1}}^{-} \otimes D_{1} \otimes I_{\bar{V} M}, & m=1, \\
I_{k_{2}}^{-} \otimes D_{1} \otimes I_{\bar{V} M}, & m>1,\end{cases} \\
& Q_{i, i+1}=\left(\begin{array}{cc}
Q_{i, i+1}^{(0,0)} & Q_{i, i+1}^{(0,1)} \\
O & Q_{i, i+1}^{(1,1)}
\end{array}\right), \quad i>0, \\
& Q_{i, i+1}^{(0,0)}= \begin{cases}I_{k_{2}+1} \otimes D_{1} \otimes I_{\bar{V} M}, & i<m-1, \\
I_{k_{2}, k_{1}} \otimes D_{1} \otimes I_{\bar{V} M}, & i=m-1, \\
I_{k_{1}+1} \otimes D_{1} \otimes I_{\bar{V} M}, & i>m-1,\end{cases} \\
& Q_{i, i+1}^{(0,1)}=\delta_{i=m-1} \tilde{I} \otimes D_{1} \otimes I_{\bar{V} M}, \\
& Q_{i, i+1}^{(1,1)}=I_{K+1} \otimes D_{1} \otimes I_{\bar{V} M},
\end{aligned}
$$

$$
\begin{aligned}
Q_{1,0} & =\left(\begin{array}{c}
\alpha I_{k_{1}, K} \otimes I_{\bar{W} \bar{V} M} \\
I_{(K+1) \bar{W} \bar{V}} \otimes \mathbf{S}_{\mathbf{0}} \boldsymbol{\beta}
\end{array}\right), \quad i>0, \quad m=1, \\
Q_{1,0} & =\left(\begin{array}{c}
\alpha I_{k_{2}, K} \otimes I_{\bar{W} \bar{V} M} \\
I_{(K+1) \bar{W} \bar{V}} \otimes \mathbf{S}_{\mathbf{0}} \boldsymbol{\beta}
\end{array}\right), \quad i>0, \quad m>1,
\end{aligned}
$$$$
Q_{i, i-1}=\left(\begin{array}{cc}
Q_{i, i-1}^{(0,0)} & O \\
Q_{i, i-1}^{(1,0)} & Q_{i, i-1}^{(1,1)}
\end{array}\right), \quad i>1
$$

$$
Q_{i, i-1}^{(0,0)}= \begin{cases}i \alpha I_{k_{2}+1} \otimes I_{\bar{W} \bar{V} M}, & i<m, \\ i \alpha I_{k_{1}, k_{2}} \otimes I_{\bar{W} \bar{V} M}, & i=m, \\ i \alpha I_{k_{1}+1} \otimes I_{\bar{W} \bar{V} M}, & i>m,\end{cases}
$$$$
Q_{i, i-1}^{(1,0)}= \begin{cases}\hat{I}_{k_{2}} \otimes I_{\bar{W} \bar{V}} \otimes \mathbf{S}_{\mathbf{0}} \boldsymbol{\beta}, & i \leq m, \\ \hat{I}_{k_{1}} \otimes I_{\bar{W} \bar{V}} \otimes \mathbf{S}_{\mathbf{0}} \boldsymbol{\beta}, & i>m,\end{cases}
$$

$$
\begin{aligned}
Q_{i, i-1}^{(1,1)}= & (i-1) \alpha I_{(K+1) \bar{W} \bar{V} M} \\
& +E^{-} \otimes I_{\bar{W} \bar{V}} \otimes \mathbf{S}_{\mathbf{0}} \boldsymbol{\beta} .
\end{aligned}
$$

Proof. The proof of the lemma proceeds by analysing all transitions of the Markov chain $\xi_{t}, t \geq 0$, during an interval of an infinitesimal length and rewriting the intensities of these transitions in block matrix form.

The negative diagonal entries of the matrix $Q_{0,0}$ define, up to the sign, the intensities of the exit of the Markov chain $\xi_{t}$ from the macro-state $(0,0)$. Such an exit can happen due to a change in the state of the underlying processes of $M A P$ arrivals of customers and energy. The non-diagonal entries of the matrix $\left(Q_{0,0}\right)$ define the intensities of the transition of the Markov chain $\xi_{t}$ inside the macro-state $(0,0)$. These transitions can happen due to the change in the state of the underlying processes of $M A P_{1}$ and $M A P_{2}$ that are not accompanied by a customer or a unit of energy arrival. This reasoning explains the summand $I_{K+1} \otimes\left(D_{0} \oplus H_{0}\right) \otimes I_{M}$ on the right-hand side of the formula for the matrix $Q_{0,0}$. The symbols of Kronecker product and sum of matrices are very helpful for description the intensities of joint transitions of several independent Markov chains. The summand $E^{+} \otimes I_{\bar{W}} \otimes H_{1} \otimes I_{M}$ on the right-hand side of formula for the matrix $Q_{0,0}$ corresponds to a possibility of transition of the Markov chain $\xi_{t}$ inside the macro-state $(0,0)$ due to the change in the state of the underlying process of $M A P_{2}$ that leads to the arrival of a unit of energy. The matrix $E^{+}$here reflects the increase in the number of units of energy in the buffer by one.

The negative diagonal entries of the matrix $Q_{i, i}^{(r, r)}, i \geq 1$, up to the sign define the intensities of 
the exit of the Markov chain $\xi_{t}$ from the macro-state $(i, r), r=0,1$. This exit can happen due to customer service completion, departure of a customer from the buffer due to impatience, and also the change in the state of the underlying processes of $M A P$ arrivals of customers and energy. The non-diagonal entries of the matrix $\left(Q_{i, i}\right)^{(r, r)}$ define the intensities of the transition of the Markov chain $\xi_{t}$ inside the macro-state $(i, r)$. These transitions can happen due to the change of the state of the underlying process of $M A P_{1}$ that in not accompanied by a customer arrival or any change in the state of the underlying process of $M A P_{2}$, including the change that causes the arrival of an energy unit when this arrival does not lead to switching the server on.

The zero block in the structure (1) of the matrix $Q_{i, i}, i \geq 1$, stems from the fact that the transition from the macro-state $(i, 1)$ to the macro-state $(i, 0)$ is not possible because the server can be switched off only during the epoch of service completion which mandatorily leads to a decrease in the number of customers in the system. The matrices $\left(Q_{i, i}\right)^{(0,1)}$ define the intensities of switching the server on caused by energy unit arrival. In this case the number of energy units decreases by $n$ and the service process starts.

The matrix $Q_{0,1}$ defines the intensities of the events that lead to the increase of the number of customers in the system from zero to one. In this case, the server can be switched on (the intensities of this event are defined by the matrix $Q_{0,1}^{(0,1)}$ ) or remain in the switched off mode (these intensities are defined by the matrix $Q_{0,1}^{(0,0)}$ ).

The matrix $Q_{i, i+1}, i \geq 1$, defines the intensities of events that lead to an increase in the number of customers in the system and has the form (2). This form is explained by the fact that the arrival of a customer cannot lead to switching the server off. So, the matrix $Q_{i, i+1}^{(1,0)}, i \geq 1$, has all zero entries. The blocks $Q_{i, i+1}^{(r, r)}, i \geq 1, r=0,1$, define the intensities of customers arrival that do not change the state of the server. The block $Q_{i, i+1}^{(0,1)}, i \geq 1$, defines the intensities of customers arrival during the epoch when the server is switched off; $m-1$ customers are staying in the buffer and the number of energy units is greater than $k_{1}$. In this case, the server is switched on, $n+1$ energy units disappear and a new service starts.

The matrix $Q_{1,0}$ defines the intensities of events that lead to the decrease in the number customers in the system from one to zero. This can happen if the server is switched on, service of a customer is finished and the server switches off (these intensities are defined by the matrix $\left.I_{(K+1) \bar{W} \bar{V}} \otimes \mathbf{S}_{\mathbf{0}} \boldsymbol{\beta}\right)$, or if the server is switched off and the customer leaves the buffer due to impatience (these intensities are defined by the matrix $\left.\alpha I_{\delta_{i=m} k_{1}+\left(1-\delta_{i=m}\right) k_{2}, K} \otimes I_{\bar{W} \bar{V} M}\right)$.

The matrix $Q_{i, i-1}, i \geq 2$, defines the intensities of events that lead to the decrease in the number of customers in the system by one and has the form (3). The blocks $Q_{i, i-1}^{(0,1)}, i \geq 2$, are equal to zero because the decrease in the number of customers in the system cannot cause the switching the server. The block $\left(Q_{i, i-1}\right)^{(0,0)}$ defines the intensities of customers leaving the buffer due to impatience. The block $\left(Q_{i, i-1}\right)^{(1,1)}$ defines the intensities of the event that the service of a customer is finished and a customer from the buffer is chosen for service or a customer leaves the buffer due to impatience. The block $\left(Q_{i, i-1}\right)^{(1,0)}$ defines the intensity of the event that in the service completion epoch the buffer of energy is empty and the server is switched off.

Since no more than one customer can leave the system and no more than one customer can enter the system during an interval of infinitesimal length, the matrices $Q_{i, j}, i, j \geq 0$ are zero matrices when $|i-j|>1$.

Corollary 1. The Markov chain $\xi_{t}, t \geq 0$, belongs to the class of continuous-time asymptotically quasi-Toeplitz Markov chains (AQTMC) (see Klimenok and Dudin, 2006).

Proof. Let us introduce the matrix $R_{i}, i>m$, as a diagonal one with diagonal entries which are defined as the moduli of the corresponding diagonal entries of the matrix $Q_{i, i}$. One can see that the matrix $R_{i}, i>m$, has the following form:

$$
R_{i}=\left(\begin{array}{cc}
R_{i}^{(0)} & O \\
O & R_{i}^{(1)}
\end{array}\right), \quad i>m
$$

where

$$
\begin{aligned}
& R_{i}^{(0)}=i \alpha I+I_{k_{1}+1} \otimes(\Sigma \oplus \Delta) \otimes I_{M}, \\
& R_{i}^{(1)}=I_{K+1} \otimes(\Sigma \oplus \Delta \oplus \tilde{S})+(i-1) \alpha I .
\end{aligned}
$$

Here $\Sigma, \Delta$ and $\tilde{S}$ are diagonal matrices whose diagonal entries are defined as the corresponding diagonal entries of the matrices $-D_{0},-H_{0}$ and $-S$, respectively.

It is easy to verify that the following limits exist:

$$
\begin{gathered}
Y_{0}=\lim _{i \rightarrow \infty} R_{i}^{-1} Q_{i, i-1}, \quad Y_{1}=\lim _{i \rightarrow \infty} R_{i}^{-1} Q_{i, i}+I, \\
Y_{2}=\lim _{i \rightarrow \infty} R_{i}^{-1} Q_{i, i+1} .
\end{gathered}
$$

According to the definition given by Klimenok and Dudin (2006), the existence of the limits $Y_{0}, Y_{1}$ and $Y_{2}$ means that the Markov chain $\xi_{t}, t \geq 0$, belongs to the class of continuous-time asymptotically quasi-Toeplitz Markov chains ( $A Q T M C)$.

Let us analyze the properties of this Markov chain. This analysis should include derivation of conditions, which should be imposed on the system parameters to guarantee the existence of the stationary distribution of the states of the chain (ergodicity condition), and a procedure for computation of the stationary probabilities of the states. 


\section{Ergodicity condition and the stationary probabilities of the system states}

Let us separately consider the cases when the customers are impatient $(\alpha>0)$ and absolutely patient $(\alpha=$ $0)$. In the former case, the customers, due to their impatience, can leave the system without service. In this case, as we will show below, the stationary distribution of the states of the chain exists for any set of the system parameters and the main interest is in the analysis of the customer loss probability and finding the ways for reducing this probability. In the latter case, the ergodicity condition is the most important subject of analysis. This condition answers the question whether or not the system has a stationary mode of operation under a fixed set of parameters of flows of customers and energy and the service rate. This condition is not trivial and is presented below, while the loss probability in this case is equal to zero. So, numerical analysis of the loss probability presented in Section 6 should be replaced with analysis and optimization of the average waiting or sojourn time of an arbitrary customer. This average time can be easily computed via Little's formula.

Let us first consider the case when $\alpha>0$. As follows from the work of Klimenok and Dudin (2006), a sufficient condition of the ergodicity of the Markov chain $\xi_{t}, t \geq 0$, is the fulfillment of the inequality

$$
\mathbf{y} Y_{0} \mathbf{e}>\mathbf{y} Y_{2} \mathbf{e}
$$

where the vector $\mathbf{y}$ is the unique solution to the system

$$
\mathbf{y}\left(Y_{0}+Y_{1}+Y_{2}\right)=\mathbf{y}, \quad \mathbf{y e}=1
$$

It is easy to verify that in the case considered the matrices $Y_{0}, Y_{1}$ and $Y_{2}$ have the following form:

$$
Y_{0}=I, \quad Y_{1}=O, \quad Y_{2}=O
$$

and the ergodicity condition is given by the inequality $1>0$, which is true for all possible values of the system parameters. Accordingly, the stationary distribution of the Markov chain (and the stationary regime of queueing system operation) in case $\alpha>0$ exists for any set of the system parameters.

Now, let us consider the case when $\alpha=0$. It is evident that for $i>m$ the matrices $Q_{i, i}, Q_{i, i-1}$ and $Q_{i, i+1}$ do not depend on $i$ and are defined as follows:

$$
\begin{aligned}
Q_{i, i}= & Q^{0}=\left(\begin{array}{cc}
Q^{(0,0)} & Q^{(0,1)} \\
O & Q^{(1,1)}
\end{array}\right), \\
Q^{(0,0)}= & I_{k_{1}+1} \otimes\left(D_{0} \oplus H_{0}\right) \otimes I_{M} \\
& +E_{k_{1}}^{+} \otimes I_{\bar{W}} \otimes H_{1} \otimes I_{M},
\end{aligned}
$$

$$
\begin{aligned}
Q^{(0,1)}= & E_{k_{1}}^{-} \otimes I_{\bar{W}} \otimes H_{1} \otimes I_{M}, \\
Q^{(1,1)}= & I_{K+1} \otimes\left(D_{0} \oplus H_{0} \oplus S\right) \\
& +E^{+} \otimes I_{\bar{W}} \otimes H_{1} \otimes I_{M},
\end{aligned}
$$

$$
\begin{aligned}
Q_{i, i-1}= & Q^{-} \\
= & \left(\begin{array}{cc}
O_{\left(k_{1}+1\right) \bar{W} \bar{V} M} & O \\
\hat{I}_{k_{1}} \otimes I_{\bar{W} \bar{V}} \otimes \mathbf{S}_{\mathbf{0}} \boldsymbol{\beta} & E^{-} \otimes I_{\bar{W} \bar{V}} \otimes \mathbf{S}_{\mathbf{0}} \boldsymbol{\beta}
\end{array}\right), \\
& Q_{i, i+1}=Q^{+}=I_{k_{1}+K+2} \otimes D_{1} \otimes I_{\bar{V} M} .
\end{aligned}
$$

In consequence, in the case when $\alpha=0$, the Markov chain $\xi_{t}, \quad t \geq 0$, belongs to the class of continuous-time quasi-Toeplitz Markov chains ( $Q T M C$ ) or quasi-birth-and-death processes, (see Neuts, 1981).

It follows from the work of Neuts (1981) that the necessary and sufficient ergodicity condition of the quasi-birth-and-death process is the fulfillment of the inequality

$$
\boldsymbol{y} Q^{-} \mathbf{e}>\boldsymbol{y} Q^{+} \mathbf{e}
$$

where the row vector $\boldsymbol{y}$ is the unique solution to the following system of linear algebraic equations:

$$
\boldsymbol{y}\left(Q^{-}+Q^{0}+Q^{+}\right)=\mathbf{0}, \quad \boldsymbol{y e}=1 .
$$

The ergodicity condition for the model considered is easily verified algorithmically. The finite system (5) of linear algebraic equations is solved on a computer. Then, fulfillment of the inequality (4) is checked.

If $\alpha>0$ or the condition (4) is fulfilled in the case when $\alpha=0$, then the positive stationary probabilities of the system states $\pi(i, r, k, \nu, \zeta, \eta), i \geq 0, r=\overline{0,1}, k=$ $\overline{0, K}, \nu=\overline{0, W}, \zeta=\overline{0, V}, \eta=\overline{1, M}$, exist.

Let us form the row vectors $\boldsymbol{\pi}(i, r, k)$ of these probabilities enumerated in lexicographic order of the components $\nu, \zeta, \eta$. Then, let us form the row vectors

$$
\begin{gathered}
\boldsymbol{\pi}(0,0)=(\boldsymbol{\pi}(0,0,0), \boldsymbol{\pi}(0,0,1), \ldots, \boldsymbol{\pi}(0,0, K)), \\
\boldsymbol{\pi}(i, 0)=\left(\boldsymbol{\pi}(i, 0,0), \boldsymbol{\pi}(i, 0,1), \ldots, \boldsymbol{\pi}\left(i, 0, k_{2}\right)\right), \\
i=\overline{1, m-1}, \\
\boldsymbol{\pi}(i, 0)=\left(\boldsymbol{\pi}(i, 0,0), \boldsymbol{\pi}(i, 0,1), \ldots, \boldsymbol{\pi}\left(i, 0, k_{1}\right)\right), \\
i \geq m, \\
\boldsymbol{\pi}(i, 1)=(\boldsymbol{\pi}(i, 1,0), \boldsymbol{\pi}(i, 1,1), \ldots, \boldsymbol{\pi}(i, 1, K)), \\
\quad i \geq 1, \\
\boldsymbol{\pi}_{i}=(\boldsymbol{\pi}(i, 0), \boldsymbol{\pi}(i, 1)), \quad i \geq 1 .
\end{gathered}
$$


It is well known that the probability vectors $\boldsymbol{\pi}_{i}, i \geq$ 0 , satisfy the following system of linear algebraic equations:

$$
\begin{aligned}
&\left(\boldsymbol{\pi}_{0}, \boldsymbol{\pi}_{1}, \ldots, \boldsymbol{\pi}_{i}, \ldots\right) Q=\mathbf{0}, \\
&\left(\boldsymbol{\pi}_{0}, \boldsymbol{\pi}_{1}, \ldots, \boldsymbol{\pi}_{i}, \ldots\right) \mathbf{e}=1,
\end{aligned}
$$

where $Q$ is the infinitesimal generator of the Markov chain $\xi_{t}, t \geq 0$. It is not possible to solve this system directly on a computer because the system is infinite. The well-known results for $M / G / 1$ or $G / M / 1$ type Markov chains by $M$. Neuts are not applicable here because the behavior of the Markov chain $\xi_{t}$ is state inhomogeneous and the generator $Q$ does not have a Toeplitz-like structure. To solve this system, a numerically stable algorithm that takes into account that the matrix $Q$ has a block-tridiagonal structure, which is presented by Dudina et al. (2013), can be proposed.

Theorem 2. The stationary probabilities vectors $\boldsymbol{\pi}_{i}, i \geq$ 0 , are calculated by the formula

$$
\boldsymbol{\pi}_{i}=\boldsymbol{\pi}_{0} F_{i}, \quad i \geq 1 .
$$

Here the matrices $F_{i}$ are calculated using the recursion

$$
F_{i}=-F_{i-1} Q_{i-1, i}\left(Q_{i, i}+Q_{i, i+1} G_{i}\right)^{-1}, \quad i \geq 1,
$$

with the initial condition $F_{0}=I$, where the stochastic matrices $G_{i}$ are computed using the recursion

$$
G_{i}=-\left(Q_{i+1, i+1}+Q_{i+1, i+2} G_{i+1}\right)^{-1} Q_{i+1, i}, \quad i \geq 0,
$$

and the vector $\boldsymbol{\pi}_{0}$ is the unique solution to the system of linear algebraic equations

$$
\boldsymbol{\pi}_{0}\left(Q_{0,0}+Q_{0,1} G_{0}\right)=\mathbf{0}, \quad \boldsymbol{\pi}_{0} \sum_{i=0}^{\infty} F_{i} \mathbf{e}=1 .
$$

The recursion for matrices $G_{i}, \quad i \geq 0$, is the backward one and to compute the matrix $G_{i}$ we must know all matrices $G_{l}, \quad l>i, i \geq 0$. As was proven by Klimenok and Dudin (2006), the sequence of the matrices $G_{i}$ converges, as $i$ approaches infinity, to a matrix $G$, which is the minimal non-negative solution to the non-linear matrix equation

$$
G=Y_{0}+Y_{1} G+Y_{2} G^{2} .
$$

Thus, for any predefined small positive number $\epsilon_{G}$, there exists such a value $i_{0}$ that the norm of the matrix $G_{i}-G$ is less than $\varepsilon_{G}$ for all $i, i \geq i_{0}$. Accordingly, in the backward recursion we can set $G_{i}=G$ for $i \geq i_{0}$. The matrices $F_{i}, \quad i \geq 0$, have non-negative entries and, because we assume that the chain under consideration is ergodic, the norm of the matrix $F_{i}$ tends to zero as $i$ approaches infinity. A recursive calculation of the matrices $F_{i}$ from recursion can be halted when the norm of the matrix $F_{i}$ becomes less than some preassigned positive $\epsilon_{F}$. The positive feature of the presented algorithm is its numerical stability guaranteed by the fact of operation with only non-negative matrices at all steps of the algorithm.

\section{Performance measures}

As soon as the vectors $\boldsymbol{\pi}_{i}, i \geq 0$, have been calculated, we are able to find various performance measures of the system.

The probability $P_{\text {serv }}$ that at an arbitrary moment the server is busy is computed as

$$
P_{\text {serv }}=\sum_{i=1}^{\infty} \boldsymbol{\pi}(i, 1) \mathbf{e} .
$$

The average number $N_{\text {buffer }}^{\text {(customer) }}$ of customers in the buffer is given by

$$
N_{\text {buffer }}^{\text {(customer) }}=\sum_{i=1}^{\infty} \sum_{r=0}^{1}(i-r) \boldsymbol{\pi}(i, r) \mathbf{e} .
$$

The average number $N_{\text {buffer }}^{\text {(energy }}$ of units of energy in the buffer is computed as

$$
\begin{aligned}
N_{\text {buffer }}^{\text {(energy) }}= & \left(\sum_{k=1}^{K} k \boldsymbol{\pi}(0,0, k)+\sum_{i=1}^{m-1} \sum_{k=1}^{k_{2}} k \boldsymbol{\pi}(i, 0, k)\right. \\
& +\sum_{i=m}^{\infty} \sum_{k=1}^{k_{1}} k \boldsymbol{\pi}(i, 0, k) \\
& \left.+\sum_{i=1}^{\infty} \sum_{k=1}^{K} k \boldsymbol{\pi}(i, 1, k)\right) \mathbf{e} .
\end{aligned}
$$

The average intensity $\lambda_{\text {out }}$ of the flow of customers that receive service is given by

$$
\lambda_{\text {out }}=\sum_{i=1}^{\infty} \boldsymbol{\pi}(i, 1)\left(\mathbf{e}_{(K+1) \bar{W} \bar{V}} \otimes \mathbf{S}_{\mathbf{0}}\right) .
$$

The probability $P_{\text {loss }}^{\text {(customer) }}$ that an arbitrary customer will be lost is computed as

$$
P_{\text {loss }}^{\text {(customer) }}=1-\frac{\lambda_{\text {out }}}{\lambda_{c}} .
$$

The probability $P_{\text {loss }}^{\text {(energy) }}$ that an arbitrary unit of energy will be lost is given by

$$
\begin{aligned}
& P_{\text {loss }}^{(\text {energy })} \\
& =\frac{1}{\lambda_{e}}\left(\sum_{i=1}^{\infty} \boldsymbol{\pi}(i, 1, K)+\boldsymbol{\pi}(0,0, K)\right) \\
& \quad \times\left(I_{\bar{W}} \otimes H_{1} \otimes I_{M}\right) \mathbf{e} .
\end{aligned}
$$

The probability $P_{\text {serv-idle }}$ that at in arbitrary epoch the server is idle (is switched off) is computed from

$$
P_{\text {serv-idle }}=\sum_{i=0}^{\infty} \boldsymbol{\pi}(i, 0) \mathbf{e} .
$$




\section{Numerical examples and the optimization problem}

To demonstrate the feasibility of the results and prove the importance of the consideration of the correlated arrival flows and the phase-type service time distribution, we present the following numerical example.

Let us consider a system with stationary Poisson arrivals of customers and energy and an exponential service time distribution (System 1), and the system with Markovian arrival processes of customers and energy and phase-type service time distribution (System 2) with the same average intensities.

We assume that, in the case of System 1, the arrival process of customers is defined by the matrices $D_{0}=$ $(-0.6), D_{1}=(0.6)$. This arrival flow is a stationary Poisson process and has the intensity $\lambda_{c}=0.6$, the coefficient of correlation is equal to 0 and the coefficient of variation is equal to 1 . The arrival process of energy is defined by the matrices $H_{0}=(-0.9), H_{1}=(0.9)$. This arrival flow is a stationary Poisson process and has the intensity $\lambda_{e}=0.9$, the coefficient of correlation is equal to 0 and the coefficient of variation is equal to 1 . The service time distribution is exponential and defined by $S=(-1.5)$ and $\boldsymbol{\beta}=(1)$. The average service time is equal to $\frac{2}{3}$, the coefficient of variation $c_{\mathrm{var}}$ is equal to 1 .

In the case of System 2, we assume that the arrival process of customers is defined by the matrices

$$
\begin{gathered}
D_{0}=\left(\begin{array}{cc}
-0.810984 & 0 \\
0 & -0.026322
\end{array}\right), \\
D_{1}=\left(\begin{array}{cc}
0.805592 & 0.005392 \\
0.01466 & 0.01166
\end{array}\right) .
\end{gathered}
$$

The fundamental rate of this arrival process is $\lambda_{c}=0.6$, the coefficient of correlation of successive inter-arrival times is 0.2 , the coefficient of variation of inter-arrival times is 12.34 . matrices

The arrival process of energy is defined by the

$$
\begin{aligned}
& H_{0}=\left(\begin{array}{cc}
-3.057919 & 0 \\
0.000914 & -0.0992
\end{array}\right), \\
& H_{1}=\left(\begin{array}{ll}
3.026068 & 0.031851 \\
0.010919 & 0.087367
\end{array}\right) .
\end{aligned}
$$

The fundamental rate of this arrival process is $\lambda_{e}=0.9$, the coefficient of correlation of successive inter-arrival times is 0.4 , the coefficient of variation of inter-arrival times is 12.39 .

$\mathrm{PH}$ service process of customers is characterized by the vector $\boldsymbol{\beta}=(1,0)$ and the matrix

$$
S=\left(\begin{array}{cc}
-3 & 3 \\
0 & -3
\end{array}\right)
$$

The mean service time in this service process is equal to $2 / 3$, the coefficient of variation $c_{\mathrm{var}}$ is equal to 0.5 .

The rest of the system parameters are the same for each system under consideration and are assumed to be as follows:

$$
K=20, \quad k_{2}=16, \quad \alpha=0.015, \quad n=2 .
$$

One of the most important performance characteristics of the model considered is the probability $P_{\text {loss }}^{\text {(customer) }}$ that an arbitrary customer will be lost. Consequently, it is reasonable to consider the problem of minimization of this probability via a suitable choice of the thresholds $m$ and $k_{1}$ defining the strategy of switching the server on.

To illustrate the behavior of the key performance measures of the system, we will vary the thresholds $m$ and $k_{1}$ of switching the server on strategy in the range $m \in[1,15]$ and $k_{1} \in\left[n+1, k_{2}\right]$.

Figure 2 illustrates the dependence of the loss probability of customers on $m$ and $k_{1}$ for the two systems considered. Here and in the sequel, the top part of a figure represents the performance measures of System 1, while the bottom part stars the performance measures of System 2 .
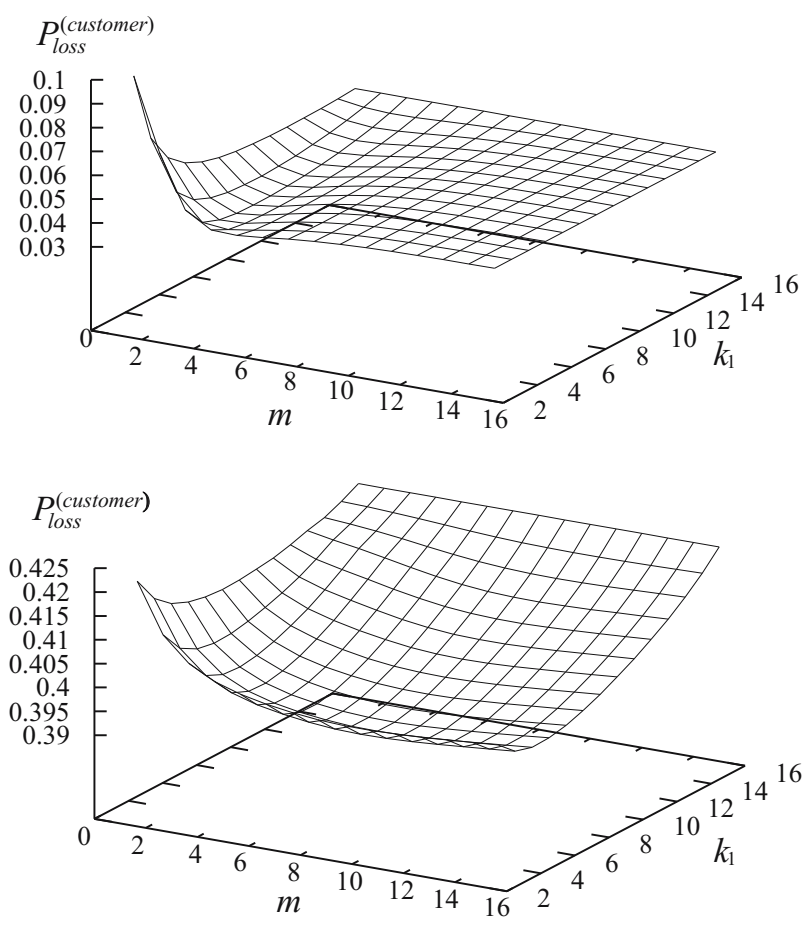

Fig. 2. Dependence of $P_{\text {loss }}^{\text {(customer) }}$ on $m$ and $k_{1}$.

The optimal values of the thresholds and loss probability for System 1 are

$$
k_{1}=7, m=3, \quad P_{\text {loss }}^{\text {(customer) }}=0.03437,
$$


and for System 2 they are

$$
k_{1}=6, m=9, P_{\text {loss }}^{\text {(customer) }}=0.39375 .
$$

The difference between the values of this probability in the cases of System 1 and System 2 is huge. The model with stationary Poisson arrival processes gives a very optimistic estimation of the loss probability. So, if the real life flow is correlated, one cannot trust results of modelling the real life system via the more simple System 1 .

Figure 3 illustrates the dependence of the probability $P_{\text {serv }}$ that the server is busy on $m$ and $k_{1}$.
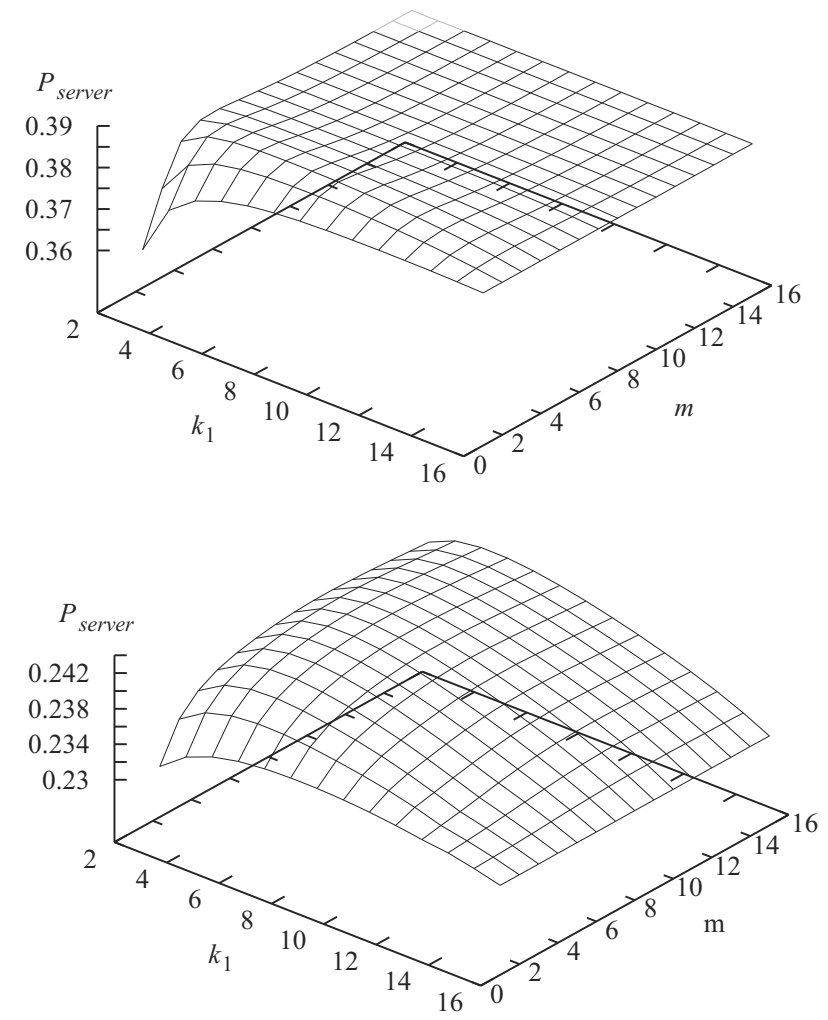

Fig. 3. Dependence $P_{\text {serv }}$ on $m$ and $k_{1}$.

The value of this probability is lower for System 2. This is understandable if we take into account Fig. 2. For System 2, the share of lost customers is much bigger than for System 1. Consequently, the server has more idle time.

Figure 4 illustrates the dependence of the probability $P_{\text {loss }}^{\text {(energ) }}$ that an arbitrary unit of energy will be lost on $m$ and $k_{1}$.

Again, similarly to Fig. 2, one can see a huge difference between the values of the probability $P_{\text {loss }}^{(\text {energy })}$ in the cases of System 1 and System 2. A lot of energy is lost in System 2 due to irregular arrival of energy units and finite capacity of the buffer. Definitely, the capacity of this buffer should be essentially increased, if possible, to get better performance of the system.
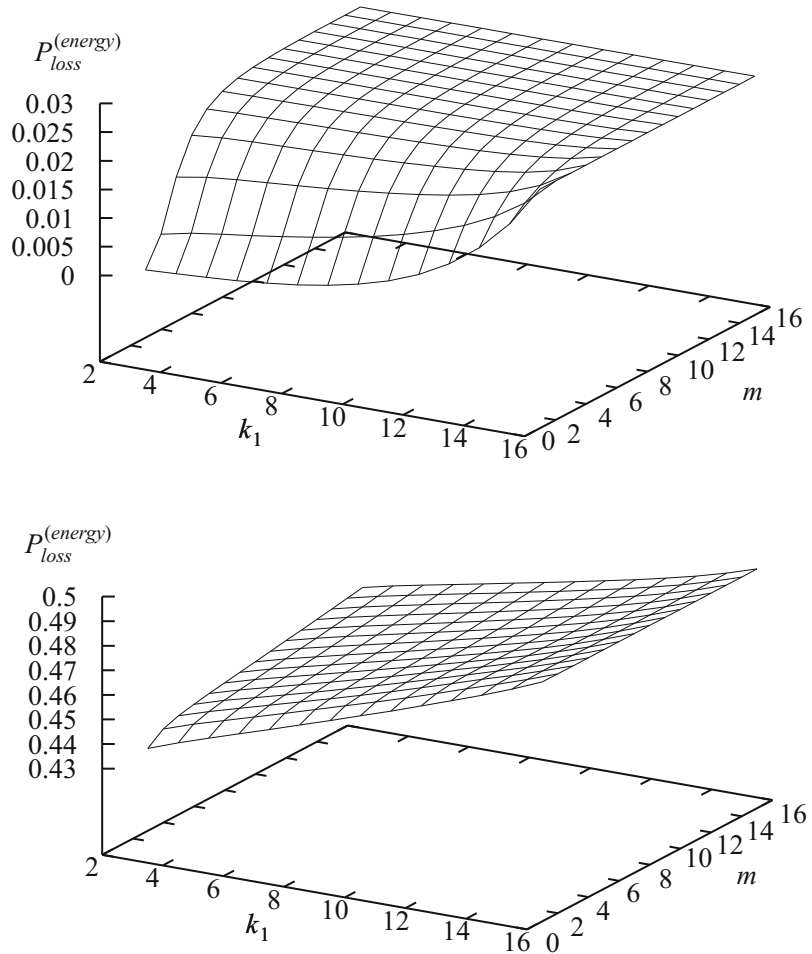

Fig. 4. Dependence of $P_{\text {loss }}^{\text {(enry) }}$ on $m$ and $k_{1}$.

Figure 5 illustrates the dependence of the average number $N_{\text {buffer }}^{\text {(energ) }}$ of units of energy in the buffer on $m$ and $k_{1}$.

This figure evidently corresponds to Fig. 4 because the higher value of probability $P_{\text {loss }}^{(\text {energy) }}$ implies, on the average, a smaller number of energy units in the buffer.

It is clear from these figures that correlation in arrival processes and the variations in the service process have a significant effect on the values of the system performance measures and optimal threshold values. Ignorance of correlation may lead to huge errors in prediction of the system performance measures and parameters of the control strategy. This explains the importance of results presented in this paper because in many potential applications the flows of customers and energy units have positive correlation of inter-arrival times. This is because the intervals when the object under observation of a sensor node is in a normal (safe) state and only a few of packets should be sent to a central node may alternate with intervals when something wrong happens and a lot of packets should be transmitted. Analogously, for the energy units arrival process, the interval when energy arrives intensively (e.g., due to sunny and (or) windy weather) alternate with intervals when energy arrives slowly (e.g., due to clouds and (or) a weak wind). 

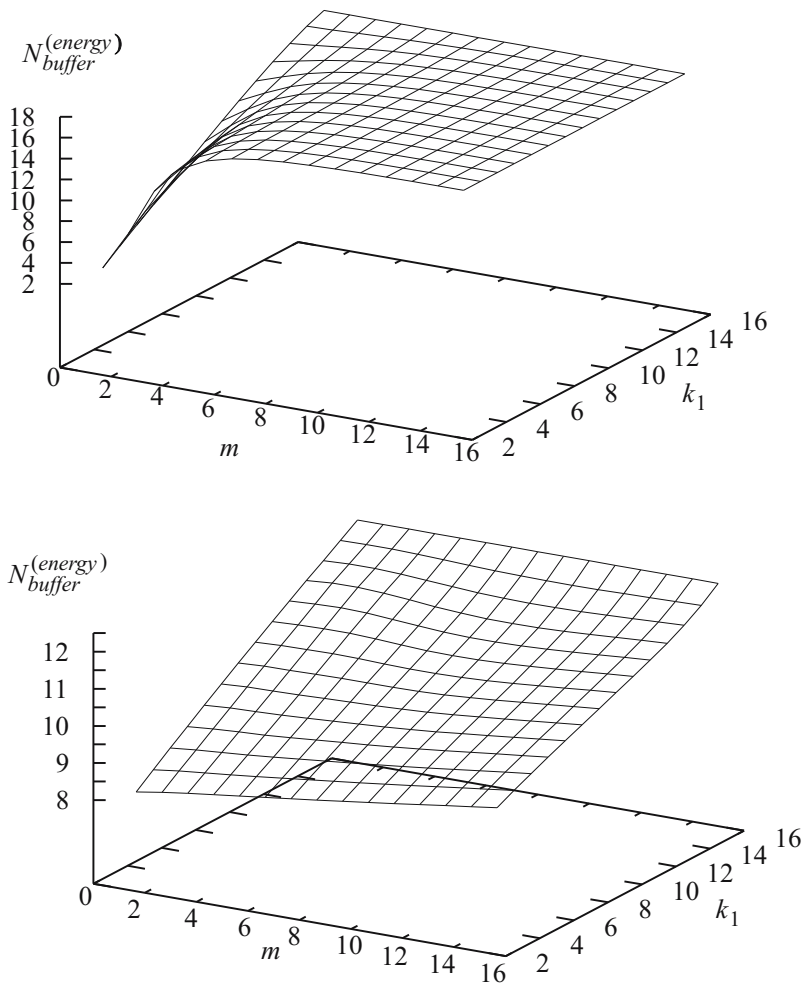

Fig. 5. Dependence of $N_{\text {buffer }}^{\text {(energy) }}$ on $m$ and $k_{1}$

\section{Conclusion}

In this paper, we considered a single-server queueing system with an infinite buffer for customers and a finite buffer for additional items (energy units) which are used for the service of customers. This model suits well, e.g., the modeling operation of a node of a sensor network which is wireless, has a battery of low capacity and harvests energy from the environment to provide transmission of packets to a central node. Customers are impatient and leave the system without service after a random amount of waiting time having an exponential distribution. This impatience models the obsolescence of information to be transmitted during the waiting time, which can make transmission of this information useless. The service of a customer, if any, is possible only in the presence of at least one unit of energy, and during this service the number of available units decreases by one. A new threshold strategy of switching the server off and on depending on the current number of customers and energy units is offered and analyzed. Numerical results show the possibility of essentially decreasing the probability of an arbitrary customer loss by means of a proper choice the thresholds, defining the strategy. The profound effect of correlation of arrival processes of customers and energy units and the variation in the service process is numerically illustrated.

Results can be used for investigating other real world systems where some non-reusable resource should be applied for the service of a customer. The results can be extended to the case of cross-correlation of arrivals of customers and energy units via consideration of the Marked Markovian arrival process (see, e.g., Kim et al., 2014). An extension to the case of a discrete time system with energy harvesting is possible by means of the technique described by Atencia (2014).

\section{Acknowledgment}

This work was supported by MEST 2015R1A2A1A05000977, NRF, Korea.

\section{References}

Akyildiz, I., Su, W., Sankarasubramaniam, Y. and Cayirci, E. (2002). Wireless sensor networks: A survey, Computer Networks 38(4): 393-422.

Atencia, I. (2014). A discrete-time system with service control and repairs, International Journal of Applied Mathematics and Computer Science 24(3): 471-484, DOI: 10.2478/amcs-2014-0035.

Doshi, B. (1986). Queueing systems with vacations-a survey, Queueing Systems 1(1): 29-66.

Dudin, A. and Klimenok, V. (1996). Queueing systems with passive servers, Journal of Applied Mathematics and Stochastic Analysis 9(2): 185-204.

Dudina, O., Kim, C. and Dudin, S. (2013). Retrial queuing system with Markovian arrival flow and phase-type service time distribution, Computers \& Industrial Engineering 66(2): 360-373.

Gelenbe, E. (2015). Synchronising energy harvesting and data packets in a wireless sensor, Energies 8(1): 356-369.

Kim, C., Dudin, A., Dudin, S. and Dudina, O. (2014). Analysis of an $M M A P / P H_{1}, P H_{2} / N / \infty$ queueing system operating in a random environment, International Journal of Applied Mathematics and Computer Science 24(3): 485-501, DOI: 10.2478/amcs-2014-0036.

Kim, C., Dudin, S. and Klimenok, V. (2009). The $M A P / P H / 1 / N$ queue with flows of customers as model for traffic control in telecommunication networks, Performance Evaluation 66(9-10): 564-579.

Klimenok, V. and Dudin, A. (2006). Multi-dimensional asymptotically quasi-Toeplitz Markov chains and their application in queueing theory, Queueing Systems 54(4): 245-259.

Mészáros, A., Papp, J. and Telek, M. (2014). Fitting traffic with discrete canonical phase type distribution and Markov arrival processes, International Journal of Applied Mathematics and Computer Science 24(3): 453-470, DOI: 10.2478/amcs-2014-0034

Neuts, M. (1981). Matrix-geometric Solutions in Stochastic Models-An Algorithmic Approach, Johns Hopkins University Press, Baltimore, MD. 
Sharma, V., Mukherji, U., Joseph, V. and Gupta, S. (2010). Optimal energy management policies for energy harvesting sensor nodes, IEEE Transactions on Wireless Communications 9(4): 1326-1336.

Takagi, H. (1991). Queueing Analysis: A Foundation of Performance Evaluation, North-Holland, Amsterdam.

Tian, N. and Zhang, Z. (2006). Vacation Queueing ModelsTheory and Applications, Springer, Heidelberg.

Tutuncuoglu, K. and Yener, A. (2012). Optimum transmission policies for battery limited energy harvesting nodes, IEEE Transactions on Wireless Communications 11(3): 1180-1189.

Yang, J. and Ulukus, S. (2012a). Optimal packet scheduling in a multiple access channel with energy harvesting transmitters, Journal of Communications and Networks 14(2): 140-150.

Yang, J. and Ulukus, S. (2012b). Optimal packet scheduling in an energy harvesting communication system, IEEE Transactions on Communications 60(1): 220-230.

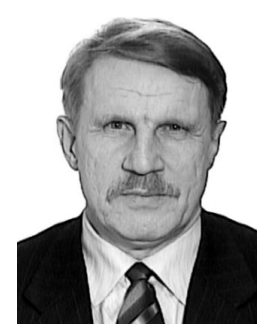

Alexander N. Dudin holds a Ph.D. degree in probability theory and mathematical statistics (1982) from Vilnius University and a D.Sc. degree (1992) from Tomsk University. He is the head of the Laboratory of Applied Probabilistic Analysis in Belarusian State University, and a professor at the Department of Probability Theory and Mathematical Statistics. He is an author of 300 publications, including more than 80 papers in top level journals. He has been the chairman of the IPC of Belarusian Winter Workshops on Queueing Theory, held since 1985. His fields of scientific interests are random processes in queueing systems, controllable queueing systems and their optimization, queueing systems in random environments, retrial queueing systems, applications of queueing theory to telecommunications.



Moon Ho Lee is a professor and former chair of the Department of Electronics Engineering and Information Engineering at Chonbuk National University, Korea. He received Ph.D. degrees from Chonnam National University, Korea, in 1984, and from the University of Tokyo, Japan in 1990 , both in electrical engineering. He was at the University of Minnesota, USA, from 1985 to 1986 as a post-doctor. He was conferred an honorary doctorate by the Bulgarian Academy of Sciences in 2010. He worked in Namyang MBC broadcasting with a chief engineer from 1970 to 1980, then he joined Chonbuk National University as a professor. Dr. Lee has made significant original contributions in the areas of mobile communication code design, channel coding, and multi-dimensional source and channel coding. He has authored 40 books, 160 SCI papers in international journals as well as 260 papers in domestic journals, and has delivered 410 presentations at international conferences. Dr. Lee is a member of the National Academy of Engineering in Korea and a foreign fellow of the Bulgarian Academy of Sciences. He is the inventor of Jacket Matrix, cited in Wikipedia over 72,250 times.

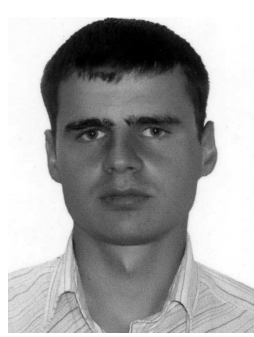

Sergey A. Dudin graduated from the Belarusian State University in 2007. In 2010, he received there a Ph.D. degree in systems analysis, control and information processing and works currently in the Research Laboratory of Applied Probabilistic Analysis. His main fields of interests are queueing systems with session arrivals and controlled tandem models.

Received: 29 June 2015

Revised: 14 September 2015 Accepted: 3 November 2015 